The AstrophysiCAL JouRNAL, 540:1084-1090, 2000 September 10

(c) 2000. The American Astronomical Society. All rights reserved. Printed in U.S.A.

\title{
APSE ALIGNMENT OF NARROW ECCENTRIC PLANETARY RINGS
}

\author{
E. I. ChiANG AND P. GoldReich \\ California Institute of Technology, Pasadena, CA 91125; echiang@tapir.caltech.edu,pmg@nicholas.gps.caltech.edu \\ Received 2000 January 10; accepted 2000 April 10
}

\begin{abstract}
The boundaries of the Uranian $\epsilon, \alpha$, and $\beta$ rings can be fitted by Keplerian ellipses. The pair of ellipses that outline a given ring share a common line of apsides. Apse alignment is surprising because the quadrupole moment of Uranus induces differential precession. We propose that rigid precession is maintained by a balance of forces due to ring self-gravity, planetary oblateness, and interparticle collisions. Collisional impulses play an especially dramatic role near ring edges. Pressure-induced accelerations are maximal near edges because there (1) velocity dispersions are enhanced by resonant satellite perturbations and (2) the surface density declines steeply. Remarkably, collisional forces felt by material in the last $\sim 100 \mathrm{~m}$ of a $\sim 10 \mathrm{~km}$ wide ring can increase equilibrium masses up to a factor of $\sim 100$. New ring surface densities are derived that accord with Voyager radio measurements. In contrast to previous models, collisionally modified self-gravity appears to allow for both negative and positive eccentricity gradients; why all narrow planetary rings exhibit positive eccentricity gradients remains an open question.

Subject headings: celestial mechanics, stellar dynamics — planets and satellites: individual (Uranus) solar system: general
\end{abstract}

\section{INTRODUCTION}

Each narrow eccentric ring surrounding Uranus is composed of particles moving on nested elliptical orbits. The outer and inner edges of a given ring define ellipses having semimajor axes $a \pm \Delta a / 2$ and eccentricities $e \pm \Delta e / 2$, where $\Delta a \ll a, \Delta e \ll e$, and $e \ll 1$. Observed values of $a, e, \Delta a$, and $\Delta e$ for the Uranian $\epsilon, \alpha$, and $\beta$ rings are listed in Table 1.

Remarkably, the set of ellipses describing an individual ring share a common line of apsides. Apse alignment is surprising because the oblateness of Uranus causes orbits of particles with different semimajor axes to precess differentially. Timescales for differential precession in the absence of other forces are extremely short; in the case of the Uranian $\epsilon$ ring, the inner edge would precess a full revolution relative to the outer edge in 175 years. Rigid precession of an eccentric planetary ring has remained a problem in ring dynamics for over 20 years.

Goldreich \& Tremaine (1979, hereafter GT) proposed that apse alignment is maintained by self-gravity. Their theory predicts that the eccentricity gradient across the ring,

$$
q_{e} \equiv a \frac{\partial e}{\partial a},
$$

must be positive. A positive eccentricity gradient in an apse-aligned ring implies that the ring is narrowest at periapse and widest at apoapse. Gravitational forces between particles are therefore greatest near periapse. Material in the inner half of the ring pulls radially inward on the outer half at periapse, generating a differential precession which exactly cancels that due to planetary oblateness.

Although the prediction that $q_{e}>0$ accords with observations of all known narrow eccentric rings, the standard selfgravity (SSG) model predicts Uranian ring masses that are too low compared to those inferred from Voyager radio occultations. Ring masses based on observations exceed predictions by factors of at least $\sim 3$ ( $\epsilon$ ring) to $\sim 50$ ( $\alpha$ and $\beta$ rings) (Tyler et al. 1986; Gresh 1990; see also the reviews by Esposito et al. 1991 and French et al. 1991). Low surface densities are particularly problematic for the $\alpha$ and $\beta$ rings. With SSG surface densities, torques exerted by inner shepherd satellites would be insufficiently strong to confine the $\alpha$ and $\beta$ rings against drag from the distended exosphere of Uranus (Goldreich \& Porco 1987, hereafter GP). In addition, as discussed by Graps et al. (1995), shapes of the $\epsilon$ ring surface density profiles as derived from occultation light curves do not accord with SSG predictions.

This paper points the way toward resolving these problems. In $\S 2$ a theory of collisionally modified self-gravity (CMSG) is qualitatively described. A simple quantitative model is set forth in $\S 3$, in which new surface density profiles are derived for the $\epsilon$ and $\alpha$ rings that are in better agreement with observations. In $\S 4$ implications of our solutions for torque balance, the role of planetary oblateness, and the value of $q_{e}$ are discussed. Directions for future research are summarized in $\S 5$.

\section{QUALITATIVE SOLUTION}

For simplicity, consider an apse-aligned eccentric ring having constant, positive $q_{e}$ across its width. The ring is filled with spherical particles of internal mass density $\rho$ and radius $r$, and the ring surface density is given by $\Sigma$. Let $n$ and $\varpi$ be the mean motion and apsidal angle, respectively, of a ring particle. Subscripts $i$ and $b$ denote quantities evaluated in the ring interior and near the ring boundary, respectively. Variables subscripted with $p$ or $s$ are associated with the central planet or shepherd satellite, respectively, and take their usual meanings. The dimensionless strength of the quadrupole moment of the planet is given by $J_{2}$. Numerical estimates in this section are made using parameters appropriate for the $\epsilon$ ring. 
TABLE 1

PARAMETERS OF ECCENTRIC URANIAN RINGS ${ }^{a}$

\begin{tabular}{lcccc}
\hline \hline Ring & $\begin{array}{c}a \\
(\mathrm{~km})\end{array}$ & $\begin{array}{c}\Delta a \\
(\mathrm{~km})\end{array}$ & $\begin{array}{c}e \\
\left(\times 10^{3}\right)\end{array}$ & $\begin{array}{c}\Delta e \\
\left(\times 10^{3}\right)\end{array}$ \\
\hline$\epsilon \ldots \ldots$ & 51,149 & 58.1 & 7.936 & 0.711 \\
$\alpha \ldots \ldots$ & 44,718 & 7.15 & 0.761 & 0.076 \\
$\beta \ldots \ldots$ & 45,661 & 8.15 & 0.442 & 0.066 \\
\hline
\end{tabular}

a Values taken from Tables I and VII of French et al. 1991.

A key ingredient missing in the SSG model is an accounting for interparticle collisions. Since ring optical depths $\tau$ measured normal to the orbital plane are typically of order unity, each particle collides, on average, a few times with its neighbors every orbital period. Only modest collisional impulses per unit mass and time, of order $0.1 \mathrm{~cm} \mathrm{~s}^{-1}$ per orbit, are required to generate differential precession rates comparable to those induced by planetary oblateness (GT). Velocity dispersions of order $c_{i} \sim 0.1$ $\mathrm{cm} \mathrm{s}^{-1}$ in the ring interior are not unreasonable: both the Keplerian shearing velocity across a particle diameter, $\sim 3 \mathrm{nr}$, and the escape velocity from the particle surface, $\sim r(8 G \rho)^{1 / 2}$, are of that order for the meter-sized bodies that plausibly compose the ring.

Although a single collision can impart an impulse of dynamically significant magnitude, multiple collisions experienced by a particle in the ring interior leave its precession rate largely unaltered. A particle in the ring interior is struck by its inside neighbors about as frequently and as forcefully as by its outside ones. Differential precession across the ring induced by smooth internal pressure gradients occurs on timescales of order $2 \pi \Sigma n a e /|\nabla P| \sim 2 \pi n a e \Delta a / c_{i}^{2} \sim 10^{6}\left(0.1 \mathrm{~cm} \mathrm{~s}^{-1} / c_{i}\right)^{2} \mathrm{yr}$, much longer than misalignment timescales set by planetary oblateness (see GT). Here the height-integrated pressure $P \sim \Sigma c_{i}^{2}$ is taken to vary over a length scale $\Delta a$.

Conditions are dramatically different near ring edges. Pressure-induced accelerations are maximal there because (1) velocity dispersions are enhanced by resonant satellite perturbations and (2) the surface density declines steeply (Borderies, Goldreich, $\&$ Tremaine 1982). The velocity dispersion near the ring boundary could be as high as

$$
c_{b} \sim \sqrt{\frac{d}{w_{r}}} c_{i} \sim 3 \frac{c_{i}}{0.1 \mathrm{~cm} \mathrm{~s}^{-1}} \mathrm{~cm} \mathrm{~s}^{-1}
$$

where $d \sim 10^{3} \mathrm{~km}$ is the ring-satellite separation, and $w_{r}$ is the width of the annulus perturbed by the satellite. To order of magnitude, the latter is given by $w_{r} \sim a\left(M_{s} / M_{p}\right)^{1 / 2} \sim 1 \mathrm{~km}$, the distance from the resonant edge at which nested periodic orbits cross. Equation (2) is derived by equating the rate of energy dissipation by collisions in the perturbed zone, $\sim \pi \Sigma a w_{r} c_{b}^{2} n \tau$, to the rate of energy deposition by the satellite, $\sim 3 n T d / 2 a \sim 9 \pi \Sigma n a c_{i}^{2} d / 2$, where $T$ is the satellite-induced confining torque whose magnitude equals that of the viscous torque, $\sim 3 \pi \Sigma c_{i}^{2} a^{2}$, in steady state.

A particle on the ring edge experiences a radially directed, collisional acceleration

$$
C \sim-\frac{\nabla P}{\Sigma} \sim \pm \frac{c_{b}^{2}}{\lambda} \hat{\boldsymbol{r}} \sim \pm c_{b} n \hat{r}
$$

where the upper (lower) sign applies to the outer (inner) ring edge. Here $P \sim \Sigma c_{b}^{2}$ is taken to vary over a radial length scale, $\lambda$, of the order of the local ring thickness, $c_{b} / n$. In a $q_{e}>0$ ring, collision rates are highest near periapse. At the periapsis of a ring boundary, the radial acceleration, $C$, generates a differential precession rate, $\Delta\langle d \varpi / d t\rangle_{C} \approx-C /$ nae, relative to the precession rate at the ring midline. This collision-induced rate is greater than the local differential rate due to planetary oblateness, $\Delta\langle d \varpi / d t\rangle_{o}$, by a substantial factor:

$$
\frac{\Delta\langle d \varpi / d t\rangle_{C}}{\Delta\langle d \varpi / d t\rangle_{o}} \sim \frac{\mp c_{b} / a e}{\mp(21 \Delta a / 8 a) J_{2} n\left(R_{p} / a\right)^{2}} \sim 40 \frac{c_{b}}{1 \mathrm{~cm} \mathrm{~s}^{-1}} .
$$

Self-gravity maintains apse alignment against differential precession caused by planetary oblateness and interparticle collisions. For self-gravity to enforce rigid precession near ring edges, surface densities there must be higher than those predicted by SSG. At ring boundaries, self-gravitational attraction must balance the extra repulsive acceleration due to collisions. To estimate the surface density near the edge, $\Sigma_{b}$, equate the collisional acceleration, $C \sim c_{b}^{2} / \lambda$, to the gravitational acceleration from a wire of linear mass density $\Sigma_{b} \lambda$ located a distance $\lambda$ away:

$$
c_{b}^{2} / \lambda \sim 2 G \Sigma_{b} .
$$

Take $\lambda=c_{b} / n \sim 50 \mathrm{~m}$ to obtain

$$
\Sigma_{b} \sim \frac{c_{b} n}{2 G} \sim 10^{3} \frac{c_{b}}{1 \mathrm{~cm} \mathrm{~s}^{-1}} \mathrm{~g} \mathrm{~cm}^{-2},
$$

which is greater than corresponding SSG predictions by factors $\gtrsim 40$. Equation (6) is equivalent to the condition that Toomre's $Q$ be of order unity at the edge. 

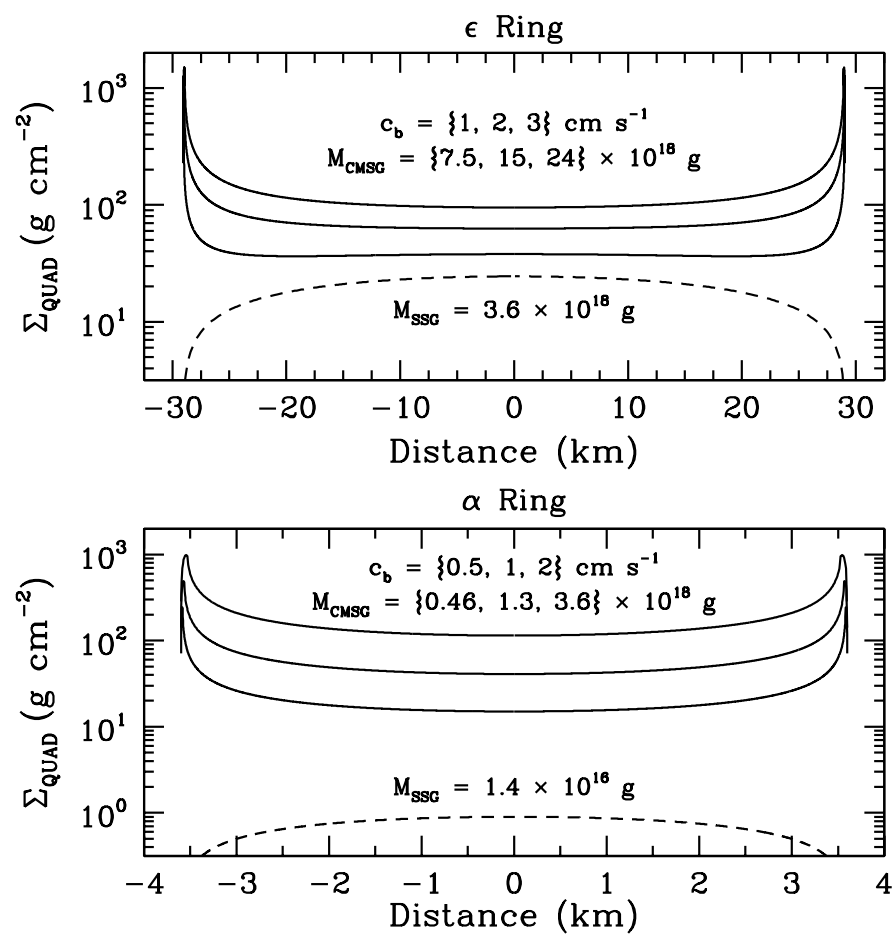

FIG. 1.-Surface density profiles at quadrature for rings $\epsilon($ top $)$ and $\alpha$ (bottom) in CMSG $\left(c_{b} \neq 0\right.$; solid line $)$ vs. SSG $\left(c_{b}=0\right.$; dashed line $)$ models. When the total CMSG ring mass, $M_{\mathrm{CMSG}}$, greatly exceeds the total SSG ring mass, $M_{\mathrm{SSG}}$, it is found empirically that $M_{\mathrm{CMSG}} \propto c_{b}^{2} / \lambda^{1 / 2} \propto c_{b}^{3 / 2}$.

These end wires of mass $\sim 2 \pi a \Sigma_{b} \lambda=\pi c_{b}^{2} a / G$ constitute new boundary conditions not found in SSG. Gravitational forces from massive end wires induce substantial differential precession in the ring interior. For self-gravity to maintain apse alignment in the interior, surface densities there must also be greater than those predicted by SSG.

\section{QUANTITATIVE MODEL}

Divide the region occupied by an apse-aligned, constant $q_{e}=a \Delta e / \Delta a$ ring into an even number $N$ of equally spaced intervals. The center of the $j$ th interval contains an elliptical wire having mass $m_{j}$, semimajor axis $a_{j}=a+$ $[j-(N+1) / 2] \Delta a / N$, and eccentricity $e_{j}=e+[j-(N+1) / 2] \Delta e / N$. Denote by $\Delta_{j}\langle d \varpi / d t\rangle$ the precession rate of the $j$ th wire relative to the precession rate of a test particle at the ring midline. Uniform precession requires

$$
\Delta_{j}\left\langle\frac{d \varpi}{d t}\right\rangle=\Delta_{j}\left(\left\langle\frac{d \varpi}{d t}\right\rangle_{o}+\left\langle\frac{d \varpi}{d t}\right\rangle_{G}+\left\langle\frac{d \varpi}{d t}\right\rangle_{c}\right)=0 .
$$

Subscripts $O, G$, and $C$ denote contributions from planetary oblateness, self-gravity, and interparticle collisions, respectively. The first two terms on the right-hand side are given by

$$
\Delta_{j}\left\langle\frac{d \varpi}{d t}\right\rangle_{o}=-\frac{21}{4} J_{2} n\left(\frac{R_{p}}{a}\right)^{2} \frac{a_{j}-a}{a}
$$

and

$$
\Delta_{j}\left\langle\frac{d \varpi}{d t}\right\rangle_{G}=\frac{q_{e} H\left(q_{e}^{2}\right)}{\pi e} n \frac{a}{M_{p}} \sum_{k \neq j} \frac{m_{k}}{a_{j}-a_{k}},
$$

where

$$
H\left(q_{e}^{2}\right) \equiv \frac{1-\sqrt{1-q_{e}^{2}}}{q_{e}^{2} \sqrt{1-q_{e}^{2}}}
$$

(see GT). For $\Delta_{j}\langle d \varpi / d t\rangle_{C}$, the following simplistic prescription is adopted:

$$
\Delta_{j}\left\langle\frac{d \varpi}{d t}\right\rangle_{c}= \begin{cases}+\left[q_{e} H\left(q_{e}^{2}\right) c_{b}^{2} / \lambda n a e\right](1-x / \lambda) & \text { if } x \equiv(j-1 / 2) \Delta a / N<\lambda, \\ -\left[q_{e} H\left(q_{e}^{2}\right) c_{b}^{2} / \lambda n a e\right](1-y / \lambda) & \text { if } y \equiv(N-j+1 / 2) \Delta a / N<\lambda, \\ 0 & \text { otherwise }\end{cases}
$$


Thus, $\Delta_{j}\langle d \varpi / d t\rangle_{C}$ is nonzero only within intervals $\delta a=\lambda$ from each edge; there, its magnitude rises linearly from zero to $q_{e} H\left(q_{e}^{2}\right) c_{b}^{2} / \lambda n a e$. As a crude justification for this maximum value, approximate the collisional acceleration as

$$
\begin{aligned}
C & \approx-\frac{\nabla P}{\Sigma} \\
& \approx \pm \frac{c_{b}^{2}}{\lambda\left(1-q_{e} \cos f\right)} \hat{\boldsymbol{r}},
\end{aligned}
$$

where the upper (lower) sign applies to the outer (inner) edge. Here the pressure gradient is taken to vary inversely as the separation between streamlines. Insert $C$ into Gauss's perturbation equation for $d \varpi / d t$ and average over true anomaly:

$$
\begin{aligned}
\left\langle\frac{d \varpi}{d t}\right\rangle & =-\frac{1}{\pi n a e} \int_{0}^{\pi} C \cos f d f \\
& =\mp \frac{q_{e} H\left(q_{e}^{2}\right) c_{b}^{2}}{\lambda n a e} .
\end{aligned}
$$

For the constant $q_{e}$ ring models presented here,

$$
\lambda=c_{b} / n,
$$

so that the only remaining free parameter is $c_{b}$. Equation (13) is relaxed for $\$ \$$ 4.2.2 and 4.3.2.

Note that this prescription for $\Delta_{j}\langle d \varpi / d t\rangle_{C}$ ignores the decrease in velocity dispersion from $c_{b}$ at the ring edge to $c_{i}$ in the ring interior. The decline in velocity dispersion occurs over a length scale of order $w_{r}$. This length scale is large compared to $c_{b} / n$ so that the gradient of velocity dispersion does not give rise to a significant radial acceleration.

For a given value of $c_{b}$, equations (7), (8), (9), (10), and (13) comprise $N$ equations in $N$ unknowns $\left\{m_{j}\right\} .{ }^{1}$ Solutions for surface density profiles at quadrature for various values of $c_{b}$ are displayed in Figure 1, for parameters appropriate to the $\epsilon$ and $\alpha$ rings; models for the $\beta$ ring are nearly identical to those of the $\alpha$ ring. In CMSG models, higher surface densities near ring edges are evident, as are higher total ring masses.

\section{DISCUSSION}

\subsection{Surface Density Profiles and Torque Balance}

Simple CMSG models, while not fully realistic, demonstrate the existence of a new class of self-gravity solution, that obtained by accounting for the modification of ring boundary conditions by interparticle collisions. Remarkably, forces felt by material in the last $\sim 100 \mathrm{~m}$ of a $\sim 10 \mathrm{~km}$ wide ring can increase equilibrium masses by factors up to 100 . The information that ring edges necessarily possess large surface densities to balance local collisional stresses is propagated into the ring interior by self-gravity.

Large S-band opacities measured by Voyager, which are incompatible with SSG surface densities (see, e.g., the review by Esposito et al. 1991), can be reconciled with average CMSG surface densities of $\sim 75-100 \mathrm{~g} \mathrm{~cm}^{-2}$ for the $\epsilon$, $\alpha$, and $\beta$ rings. Moreover, CMSG models predict that surface densities near ring edges are higher than those in the interior. This behavior is reminiscent of the "double-dip" structure seen in occultation light curves for the $\epsilon$ and $\alpha$ rings (see, e.g., the review by French et al. 1991).

Greater ring masses as implied by CMSG resolve problems associated with exospheric drag that were pointed out by GP for rings $\alpha$ and $\beta$. For the remainder of this subsection, numerical estimates will be made for the $\alpha$ ring; similar conclusions hold for the $\beta$ and $\epsilon$ rings. Surface densities are scaled to a typical CMSG value in the ring interior of $\Sigma=75 \mathrm{~g} \mathrm{~cm}^{-2}$. An inner shepherd satellite exerts a repulsive, nonlinear torque at first-order Lindblad resonances of magnitude

$$
T_{\mathrm{n} 1}^{\mathrm{L}} \approx \frac{10 \rho_{s} R_{s}^{3} \Sigma^{2} n^{2} a^{7}}{M_{p}^{2} d} \approx 6 \times 10^{17}\left(\frac{\Sigma}{75 \mathrm{~g} \mathrm{~cm}^{-2}}\right)^{2}\left(\frac{R_{s}}{10 \mathrm{~km}}\right)^{3}\left(\frac{\rho_{s}}{1.5 \mathrm{~g} \mathrm{~cm}^{-3}}\right)\left(\frac{500 \mathrm{~km}}{d}\right) \mathrm{ergs},
$$

where the satellite radius, $R_{s}$, is scaled to the Voyager upper limit of $10 \mathrm{~km}$ (Smith et al. 1986). The shepherding torque exceeds the magnitude of the drag torque exerted by the Uranian exosphere,

$$
T_{d} \approx-4 \pi m_{\mathrm{H}} n_{\mathrm{H}} v_{T} n a^{3} \Delta a \approx-4 \times 10^{16}\left(\frac{n_{\mathrm{H}}}{10^{3} \mathrm{~cm}^{-3}}\right) \mathrm{ergs} .
$$

Here $n_{\mathrm{H}}=7 \times 10^{-6} e^{32.4 R_{p} / a} \mathrm{~cm}^{-3}$ is the number density of hydrogen atoms of mass $m_{\mathrm{H}}$ in the exosphere, and $v_{T} \approx 1 \mathrm{~km} \mathrm{~s}$ is their thermal speed normal to the orbital plane (Broadfoot et al. 1986). That $T_{\mathrm{nl}}^{\mathrm{L}}>\left|T_{d}\right|$ ensures that the inner shepherd prevents ring particles from spiraling in toward Uranus.

Estimates of viscous torques $T_{v}$ also require revision. For a ring undergoing Keplerian shear, with minimum kinematic viscosity $n(\Sigma / \rho)^{2}$, the viscous torque is given by

$$
T_{v} \approx \frac{3 \pi n^{2} \Sigma^{3} a^{2}}{\rho^{2}} \approx 2.5 \times 10^{18}\left(\frac{\Sigma}{75 \mathrm{~g} \mathrm{~cm}^{-2}}\right)^{3}\left(\frac{1.5 \mathrm{~g} \mathrm{~cm}^{-3}}{\rho}\right)^{2} \mathrm{ergs}
$$

\footnotetext{
${ }^{1}$ Reflection symmetry about the ring midline reduces the number of equations necessary to $N / 2$. Typically $N \gtrsim 2000$ wires are needed to converge to within $10 \%$ of the solution for $N \rightarrow \infty$.
} 
(GP). The fact that $T_{v} \gg\left|T_{d}\right|$ ensures that ring particles on the outer edge press against the inner Lindblad resonance established by the outer shepherd.

Conclusions drawn from comparisons between $T_{\mathrm{n} 1}^{\mathrm{L}}$ and $T_{v}$ are on less sure footing. For the choice of scaling parameters, the latter exceeds the former, contrary to the requirement of the standard theory of shepherding that the torques be equal. This might be construed as evidence that the angular momentum luminosity in the ring interior is reduced below $T_{v}$ by the non-Keplerian shear associated with a nonzero $q_{e}$ (Borderies et al. 1982; GP). However, the numerical estimates for the two torques differ only by a factor of a few. The shepherding torque should be evaluated using surface densities near the edge, which CMSG predicts are higher than those in the interior; this would increase the estimate of $T_{\mathrm{n} 1}^{\mathrm{L}}$. Uncertainties in the choice of parameters preclude drawing any conclusion other than that these torques are of the same order of magnitude.

\subsection{Relative Importance of Planetary Oblateness}

$$
\text { 4.2.1. } J_{2}=0 \text { versus } J_{2} \neq 0
$$

What does CMSG predict if $J_{2}=0$ ? Figure $2 a$ displays the answer for the $\epsilon$ ring, for $c_{b}=2$ and $3 \mathrm{~cm} \mathrm{~s}^{-1}$. In contrast to SSG, a nonvanishing equilibrium surface density does not require a finite planetary oblateness; self-gravity can be balanced entirely by collisional pressure gradients. For the $\alpha$ and $\beta$ rings, solutions with and without $J_{2}$ are practically indistinguishable for $c_{b} \geq 0.5 \mathrm{~cm} \mathrm{~s}^{-1}$. The influence of $J_{2}$ on the equilibrium solution diminishes as $\Delta a$ decreases or as $c_{b}$ increases.

\subsubsection{Empirical Scaling Relations for $\mathrm{J}_{2}=0$}

For $J_{2}=0$ and fixed ring geometry, the surface density at quadrature near a given edge scales as

$$
\Sigma_{b}(0 \leq|x| \lesssim \lambda)=\frac{c_{b}^{2}}{G \lambda} f(|x| / \lambda),
$$

where $|x|$ measures distance from the edge, $c_{b}$ and $\lambda$ are the same free parameters as in equation (10), and $f$ is a dimensionless function of the similarity variable $|x| / \lambda$. Well away from ring edges, the surface density at quadrature scales as

$$
\Sigma_{i}(|x| \gg \lambda)=\frac{c_{b}^{2}}{G \sqrt{\lambda \Delta a}} g(|x| / \Delta a),
$$

where $g$ is another dimensionless function. The total ring mass scales as

$$
M \sim \frac{c_{b}^{2} a}{G}\left(\frac{\Delta a}{\lambda}\right)^{1 / 2} .
$$

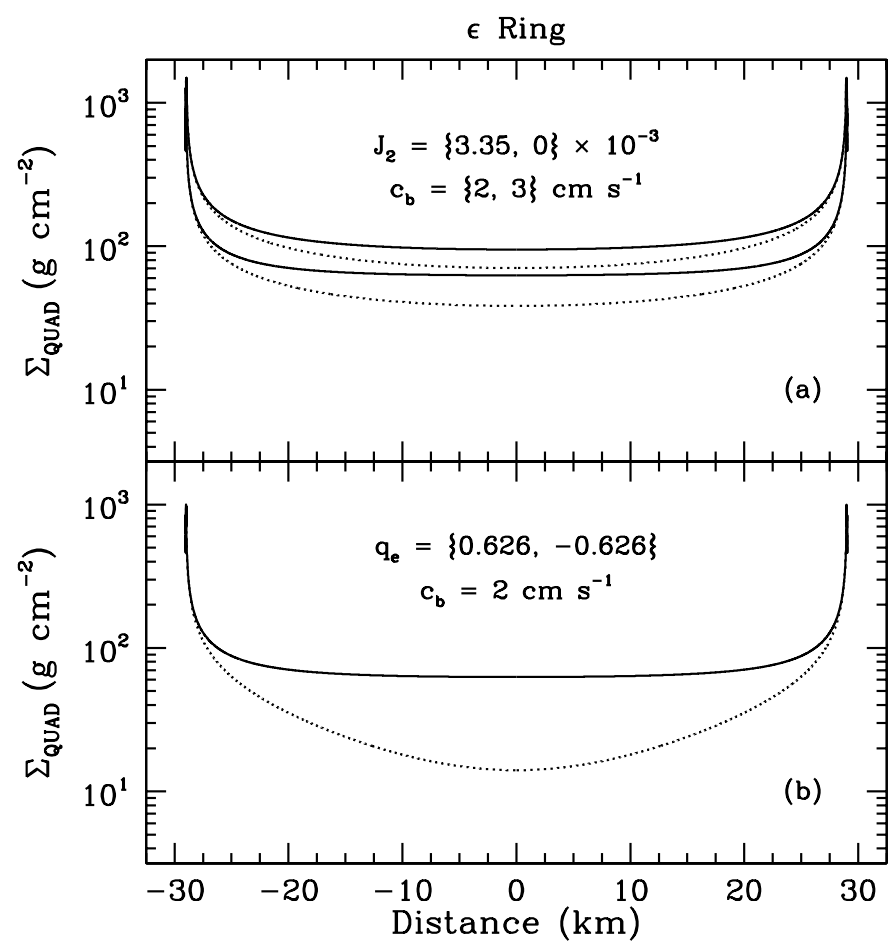

FIG. 2.-(a) CMSG $\epsilon$ ring models for which $J_{2}$ is reduced from its nominal value of $3.35 \times 10^{-3}$ (Elliot \& Nicholson 1984; solid line) to zero (dotted line). As $c_{b}$ is increased from $2 \mathrm{~cm} \mathrm{~s}^{-1}$ (lower two curves) to $3 \mathrm{~cm} \mathrm{~s}^{-1}$ (upper two curves), the influence of $J_{2}$ diminishes. For the $\alpha$ and $\beta$ rings, CMSG models with and without $J_{2}$ are practically indistinguishable for $c_{b} \geq 0.5 \mathrm{~cm} \mathrm{~s}^{-1}$ (data not shown). (b) CMSG $\epsilon$ ring models for which $q_{e}= \pm 0.626$. Contrary to SSG models, a positive $q_{e}$ is not required to obtain an equilibrium solution. 


\subsection{Value of $q_{e}$ \\ 4.3.1. Sign of $q_{e}$}

Figure $2 b$ displays a CMSG model for the $\epsilon$ ring obtained by reversing the sign of $q_{e}$. In contrast to SSG, a positive eccentricity gradient is not necessary in CMSG to obtain an equilibrium solution. This resurrects the problem of why all known eccentric planetary rings, including the Titan and Maxwell ringlets around Saturn, are narrowest at periapse and widest at apoapse.

It is possible that equilibria obtained using $q_{e}<0$ are unstable. To address this issue, a preliminary investigation of ring stability for an $N=4$ ringlet model has been undertaken. Forces due to pressure gradients are included only for the first and fourth ringlets. Collisional accelerations are treated as if they arise from anti-self-gravity forces (self-gravity with the sign of the acceleration reversed); i.e., collisional shear stresses are ignored. In this crude approximation, equilibria are found to be stable regardless of the sign of $q_{e}$; small deviations from equilibrium masses result in apsidal librations (Borderies, Goldreich, \& Tremaine 1983). It remains to be seen whether collisional shear stresses alter stability properties.

Another possibility is that initial conditions set the sign of $q_{e}$. If the ring were initially uniform in width as a function of azimuth, then planetary oblateness would determine the initial sense of differential precession within the ring. The resultant narrowing of the ring width near a true anomaly of $f=-\pi / 2$ would cause a positive eccentricity gradient to grow by self-gravity. Under this hypothesis, an $N=2$ ringlet model incorporating forces from self-gravity and planetary oblateness yields the following time evolution for the apse and eccentricity differences between outer and inner ringlets:

$$
\begin{gathered}
\delta \varpi=-A \sin \Omega_{\mathrm{lib}} t, \\
\delta e=A e\left(1-\cos \Omega_{\mathrm{lib}} t\right),
\end{gathered}
$$

where $A>0$ and $\Omega_{\text {lib }}$ are the amplitude and frequency, respectively, of libration (cf. Borderies et al. 1983). Note that the time average of $\delta e$ is positive. Inelastic collisions would damp librations, and the ring would eventually settle into an equilibrium for which $q_{e}>0$.

\subsubsection{Magnitude of $q_{e}$ near Ring Boundaries}

It has been assumed that the eccentricity gradient, $q_{e}$, is finite out to the last $\lambda=c_{b} / n \sim 50$ meters of ring material. A finite $q_{e}$ is necessary to generate a nonzero azimuthal average of the collisional acceleration (see eq. [12]). The simple quantitative model of $\S 3$ employed the observed value of $q_{e}$ averaged over the entire ring width. The true value over the last few hundred meters of ring material is unknown.

In the case of the best-studied $\epsilon$ ring, Graps et al. (1995) combined Voyager photopolarimeter and radio occultation measurements to infer the eccentricity gradient as a function of the semimajor axis. They found that $q_{e}$ decreases over the last $\sim 5 \mathrm{~km}$ from its nearly constant value of $\sim 0.65$ in the interior to $\sim 0.35$ near the edge. The radial resolution of their study was between 1 and $2 \mathrm{~km}$.

A decrease in $q_{e}$ toward ring boundaries is theoretically plausible. Distortions in a circular ring can be described by the change in separation, $\delta r$, between neighboring streamlines of the form

$$
\delta r \propto \cos m\left(\phi-\Omega_{\mathrm{pat}} t\right)
$$

where $\Omega_{\text {pat }}$ is the pattern speed of the distortion and $m$ is an integer. A constant $q_{e}$ ring that precesses rigidly in the quadrupole field of the central planet is equivalent to a distorted circular ring for which $m=1$ and $\Omega_{\text {pat }}=\langle d \varpi / d t\rangle_{Q}$. Resonant satellite perturbations, which enhance velocity dispersions within a distance $w_{r} \sim 1 \mathrm{~km}$ of ring edges, are characterized by much higher values of $m=2 a / 3 d \gg 1$ and $\Omega_{\text {pat }}=\Omega_{s}$. Satellite-induced disturbances might therefore reduce the local value of $q_{e}$. A decrease in $q_{e}$ over a distance $w_{r}$ near ring boundaries is roughly equivalent to setting $\lambda=w_{r}$ in equation (10). By the scaling relations in equations (18) and (19), this would reduce surface densities and total ring masses shown in Figure 1 by a factor of $\left(w_{r} n / c_{b}\right)^{1 / 2} \sim 4$

\section{DIRECTIONS FOR FUTURE RESEARCH}

This work is primarily a demonstration that interparticle collisions near ring boundaries play a crucial role in determining ring masses under the self-gravity hypothesis. Large collision-induced accelerations near ring edges engender locally large surface densities. These massive ring edges furnish new boundary conditions for the old self-gravity hypothesis. The nature of ring boundary conditions has not been calculated in rigorous detail; instead a prescription motivated by order-of-magnitude arguments is provided for collision-induced precession rates. Numerical simulations incorporating shepherd satellites will help to determine the actual three-dimensional collisional stress tensor and eccentricity gradient everywhere within the ring.

Why all narrow eccentric rings surrounding Uranus and Saturn are narrowest at periapse and widest at apoapse remains to be understood. Stability analyses incorporating collisional shear stresses may reveal that rings having $q_{e}<0$ are unstable. Alternatively, the sign of $q_{e}$ may be set by initial conditions. Scenarios for ring formation - e.g., the catastrophic disruption of a small moon - require further elucidation.

Viscous damping gives rise to small differences between apsidal angles of neighboring streamlines (Borderies et al. 1983). For a given apsidal shift of $\delta \varpi \ll 1$, the difference between the azimuth of maximum streamline separation and the azimuth of apoapse is given by the "pinch angle," $\delta \phi=\arctan (e \delta \varpi / \delta e) \gg \delta \tilde{\omega}$. The pinch angles calculated by Borderies et al. (1983) for their $N=2$ streamline models of the Uranian and Saturnian ringlets are suspect, however, because they neglect the boundary effects highlighted in the present work. A careful calculation of $\delta \phi(a)$ that incorporates viscous drag and the global effects of resonant forcing by shepherd satellites has yet to be performed. Upcoming observations of narrow Saturnian ringlets by the 
Cassini Orbiter might test the predictions of such a calculation, thereby furnishing a powerful diagnostic of stresses within ringlets.

Financial support for this research was provided by NSF grant 94-14232 and by a Caltech Kingsley Foundation Fellowship held by E. C. We thank an anonymous referee for useful comments.

Borderies, N., Goldreich, P., \& Tremaine, S. 1982, Nature, 299, 209 . 1983, AJ, 88, 1560

Broadfoot, A. L., et al. 1986, Science, 233, 74

Elliot, J. L., \& Nicholson, P. D. 1984, in Planetary Rings, ed. R. Greenberg \& A. Brahic (Tucson: Univ. Arizona Press), 25

Esposito, L.W., Brahic, A., Burns, J. A., \& Marouf, E. A. 1991, in Uranus, ed. J. T. Bergstrahl, E. D. Miner, \& M. S. Matthews (Tucson: Univ. Arizona Press), 410

French, R. G., Nicholson, P. D., Porco, C. C., \& Marouf, E. A. 1991, in Uranus, ed. J. T. Bergstrahl, E. D. Miner, \& M. S. Matthews (Tucson:

Univ. Arizona Press), 327

\section{REFERENCES}

Goldreich, P., \& Porco, C. C. 1987, AJ, 93, 730 (GP)

Goldreich, P., \& Tremaine, S. 1979, AJ, 84, 1638 (GT)

Graps, A. L., Showalter, M. R., Lissauer, J. J., \& Kary, D. M. 1995, AJ, 109, 226

Gresh, D. L. 1990, Ph.D. thesis, Stanford Univ.

Smith, B. A., et al. 1986, Science, 233, 43

Tyler, G. L., et al. 1986, Science, 233, 79 